\title{
Qualidade de Vida Urbana em Francisco Beltrão (SW/PR) por meio de Geoprocessamento e Análise Multicritério
}

\author{
Urban Quality of Life in Francisco Beltrão (SW/PR) through Geoprocessing and Multicriteria \\ Evaluation
}

Juliano Andres

Universidade Estadual do Oeste do Paraná, PR, Brasil.

\begin{abstract}
Resumo
O processo de urbanização em Francisco Beltrão foi muito acelerado no final do século XX, o que ocasionou diferentes tipos de ocupações urbanas e contrastes espaciais nas condições de vida da população. Nesse sentido, a pesquisa possui como intuito analisar a distribuição espacial da qualidade de vida na cidade de Francisco Beltrão, Paraná, com base em dados coletados em campo e secundários, usando como ferramentas o Geoprocessamento e a Análise Multicritério. As etapas metodológicas foram: 1) diagnóstico de indicadores a partir do Censo Demográfico 2010, 2) diagnóstico de indicadores por trabalho de campo e análise de distâncias, 3) diagnóstico dos riscos de alagamentos e 4) processamento de análise espacial pela somatória linear ponderada ordenada. Os resultados demonstram uma fragmentação espacial nas condições de vida da população na cidade que, considerando a formação dos bairros, possui estreita relação com os seus fatores históricos. Como conclusão, é possível afirmar que o uso do Geoprocessamento e da Análise Multicritério permitiram uma visão sistêmica da qualidade de vida, a partir da integração dos seus vários indicadores.
\end{abstract}

Palavras-chave: Qualidade de Vida, Geoprocessamento, Análise Multicritério.

\section{Abstract}

The urbanization in Francisco Beltrão was accelerated in the late twentieth century, which different types of occupations and spatial contrasts in living conditions of the population. The research has as objective to analyze the spatial distribution of the quality of life in the town of Francisco Beltrão, Paraná, based on collected and secondary data, using the methodological support the Geoprocessing and Multicriteria Evaluation. The methodological steps were: 1) diagnosis of indicators from the 2010 Census, 2) diagnostic indicators for fieldwork and analysis of distances, 3) diagnosis of the risks of flooding, 4) processing of spatial analysis by weighted linear sum ordered. The results demonstrate a spatial fragmentation in the living conditions of the population, has a close relationship with their historical factors. Conclusion, we can say that the use of Geoprocessing and Multicriteria Analysis allowed a systems view of quality of life, from the integration of its various indicators.

Keywords: Quality of Life, Geoprocessing, Multicriteria Evaluation. 


\section{Introdução}

O processo de urbanização de Francisco Beltrão pode ser considerado recente se comparado com a maior parte das cidades brasileiras, ocorrendo de forma muito acentuada entre as décadas de 1960 e 1990. Segundo dados censitários ${ }_{\llcorner}$no ano de 1960 apenas 8,99\% da população do município habitava na área urbana e em 1991, já eram 74,46\% (IBGE, 2011). Esse rápido crescimento se deve a consolidação da cidade como centro regional de comércio e serviços e pela mecanização agrícola nos municípios da região sudoeste paranaense (Mondardo, 2009). Esse aumento populacional na cidade, que pode ser considerado exorbitante, ocasionou a perda de controle pelo poder público em relação a ocupação de muitas áreas e tornou as formas de planejamento da cidade ineficientes em alguns aspectos, fatores que contribuíram para os contrastes espaciais nas condições de vida da população.

Nesse sentido, é possível citar: a) as visíveis diferenças entre bairros e entre setores da cidade nas condições de renda, de alfabetização e de moradia; b) as diferentes formas de oferta de serviços públicos como saneamento e manutenção de locais públicos (especialmente os parques); c) a concentração de alguns serviços públicos, especialmente de saúde, em uma área central da cidade dificultando o acesso dos moradores de locais periféricos; e) loteamentos habitacionais em planícies de inundação dos rios e canais de drenagem.

Neste início do século XXI, ainda há tendência de crescimento, pois a cidade concentra o comércio e os serviços de uma região com 37 municípios, bem como recentemente houve a instalação e ampliação de indústrias, foram construídos o Hospital Regional do Sudoeste e o Centro Regional de Detenção e Ressocialização, e instalaram-se cinco universidades (duas públicas e três particulares). Tudo isso, resultou em um aumento da população urbana de 23,03\% entre 2000 e 2010, acima da média nacional que foi 14,27\%. Desse modo, é de fundamental importância pesquisas sobre as condições de vida na cidade de Francisco Beltrão que possam auxiliar o poder público no seu planejamento.

Considerando esses aspectos, a pesquisa objetiva desenvolver uma avaliação espacial da qualidade de vida na cidade de Francisco Beltrão a partir de uma análise sistêmica, usando como suportes o Geoprocessamento e a Avaliação Multicritério, e dez parâmetros selecionados por meio de fundamentação teórica.

\section{Fundamentação Teórica}

A qualidade de vida pode ser compreendida como um conceito subjetivo, que resulta de um conjunto de condições de vida que podem ser diagnosticadas por meio de análise objetiva ou subjetiva. Esses dois vieses de investigação, objetividade e subjetividade, ocasionam uma certa dualidade e geram os mais diferentes conceitos, porém, especificamente nesse trabalho, foi usado o recorte teórico apresentado por Stimson e Marans (2011). Segundo os autores, a qualidade de vida pode ser analisada a partir das condições básicas ou fundamentais dos grupos de indivíduos, mensuradas a partir de dados secundários ou coletados. Ainda colocam que nesse viés objetivo, os elementos ou indicadores são abstraídos individualmente e, num segundo momento, inter-relacionados a partir de uma análise sistêmica. Nesse sentido, pressupõem o uso de modelagem conceitual para sintetizar a qualidade de vida, que na presente pesquisa é baseada nos fundamentos de Geoprocessamento apresentados por Xavier-da-Silva (2001). 
A partir dos recortes teórico (Stimson e Marans, 2011) e metodológico (Xavie-daSilva, 2001), buscou-se uma fundamentação dos parâmetros de uso mais recorrentes para avaliar a qualidade de vida, que culminou na elaboração do quadro 1 para demonstrar 15 trabalhos desenvolvidos no Brasil e Peru, além de países europeus e asiáticos. Percebe-se que o único parâmetro usado em todas as pesquisas é "educação", sendo recorrentes também "habitação", "ambiente urbano", "trabalho e renda", "saúde", "esporte e lazer" e "transporte". Os menos usados são "saneamento", cultura" e "infraestrutura".

Quadro 1- Parâmetros da qualidade de vida utilizados pelos autores.

\begin{tabular}{|c|c|c|c|c|c|c|c|c|c|c|c|c|c|c|c|c|}
\hline $\begin{array}{c}\text { Autores* } \\
\text { / Parâmetros }\end{array}$ & $\mathbf{1}$ & $\mathbf{2}$ & $\mathbf{3}$ & $\mathbf{4}$ & $\mathbf{5}$ & $\mathbf{6}$ & $\mathbf{7}$ & $\mathbf{8}$ & $\mathbf{9}$ & $\mathbf{1 0}$ & $\mathbf{1 1}$ & $\mathbf{1 2}$ & $\mathbf{1 3}$ & $\mathbf{1 4}$ & $\mathbf{1 5}$ & $\mathbf{T}^{* *}$ \\
\hline Habitação & $\mathrm{X}$ & $\mathrm{X}$ & $\mathrm{X}$ & $\mathrm{X}$ & $\mathrm{X}$ & $\mathrm{X}$ & $\mathrm{X}$ & $\mathrm{X}$ & & $\mathrm{X}$ & $\mathrm{X}$ & $\mathrm{X}$ & $\mathrm{X}$ & $\mathrm{X}$ & $\mathrm{X}$ & $\mathbf{1 4}$ \\
\hline Saneamento & & $\mathrm{X}$ & $\mathrm{X}$ & & & $\mathrm{X}$ & $\mathrm{X}$ & $\mathrm{X}$ & $\mathrm{X}$ & $\mathrm{X}$ & $\mathrm{X}$ & & & & & $\mathbf{8}$ \\
\hline Saúde & $\mathrm{X}$ & $\mathrm{X}$ & $\mathrm{X}$ & & $\mathrm{X}$ & $\mathrm{X}$ & & & & $\mathrm{X}$ & $\mathrm{X}$ & $\mathrm{X}$ & $\mathrm{X}$ & $\mathrm{X}$ & $\mathrm{X}$ & $\mathbf{1 1}$ \\
\hline Educação & $\mathrm{X}$ & $\mathrm{X}$ & $\mathrm{X}$ & $\mathrm{X}$ & $\mathrm{X}$ & $\mathrm{X}$ & $\mathrm{X}$ & $\mathrm{X}$ & $\mathrm{X}$ & $\mathrm{X}$ & $\mathrm{X}$ & $\mathrm{X}$ & $\mathrm{X}$ & $\mathrm{X}$ & $\mathrm{X}$ & $\mathbf{1 5}$ \\
\hline Transporte & $\mathrm{X}$ & $\mathrm{X}$ & $\mathrm{X}$ & & & $\mathrm{X}$ & & & & $\mathrm{X}$ & $\mathrm{X}$ & $\mathrm{X}$ & $\mathrm{X}$ & $\mathrm{X}$ & $\mathrm{X}$ & $\mathbf{1 0}$ \\
\hline Cultura & & & $\mathrm{X}$ & $\mathrm{X}$ & & & & & & $\mathrm{X}$ & $\mathrm{X}$ & $\mathrm{X}$ & $\mathrm{X}$ & $\mathrm{X}$ & $\mathrm{X}$ & $\mathbf{8}$ \\
\hline Esporte e Lazer & $\mathrm{X}$ & $\mathrm{X}$ & $\mathrm{X}$ & & $\mathrm{X}$ & $\mathrm{X}$ & & & & $\mathrm{X}$ & $\mathrm{X}$ & & $\mathrm{X}$ & $\mathrm{X}$ & $\mathrm{X}$ & $\mathbf{1 0}$ \\
\hline Infraestrutura & & & $\mathrm{X}$ & & & $\mathrm{X}$ & & & & $\mathrm{X}$ & $\mathrm{X}$ & & & & & $\mathbf{4}$ \\
\hline Ambiente Urbano & $\mathrm{X}$ & $\mathrm{X}$ & $\mathrm{X}$ & $\mathrm{X}$ & $\mathrm{X}$ & $\mathrm{X}$ & $\mathrm{X}$ & $\mathrm{X}$ & & $\mathrm{X}$ & $\mathrm{X}$ & $\mathrm{X}$ & $\mathrm{X}$ & $\mathrm{X}$ & $\mathrm{X}$ & $\mathbf{1 4}$ \\
\hline Trabalho e Renda & $\mathrm{X}$ & $\mathrm{X}$ & $\mathrm{X}$ & $\mathrm{X}$ & $\mathrm{X}$ & $\mathrm{X}$ & $\mathrm{X}$ & $\mathrm{X}$ & $\mathrm{X}$ & & $\mathrm{X}$ & $\mathrm{X}$ & $\mathrm{X}$ & & $\mathrm{X}$ & $\mathbf{1 3}$ \\
\hline Total & $\mathbf{7}$ & $\mathbf{8}$ & $\mathbf{1 0}$ & $\mathbf{5}$ & $\mathbf{6}$ & $\mathbf{9}$ & $\mathbf{5}$ & $\mathbf{5}$ & $\mathbf{3}$ & $\mathbf{9}$ & $\mathbf{1 0}$ & $\mathbf{7}$ & $\mathbf{8}$ & $\mathbf{7}$ & $\mathbf{8}$ & $\mathbf{1 0 7}$ \\
\hline
\end{tabular}

* 1 Martins (2011); 2 Arifwidodo (2012); 3 Rezvani (2013); 4 Sandru (2012); 5 Arechavala (2008); 6 Keinert et al (2009); 7 Marques (2008); 8 Mello Filho (2003); 9 Bueno (2006); 10 Majumder (2007); 11 Nahas (2009); 12 Castelli et al (2009); 13 Oktay e Rustemli (2010); 14 Álcázar e Andrade (2008); 15 Santos et al (2004). ** Total Fonte: O Autor (2014).

A partir desses parâmetros, foram revisados os indicadores usados pelos autores para representá-los, bem como aqueles disponíveis para a cidade de Francisco Beltrão. Os indicadores vistos podem ser considerados muito diversos e diretamente ligados as particularidades do recorte espacial de cada estudo, desse modo, optou-se por demonstrar aqueles estranhos ao contexto espacial de Francisco Beltrão e que foram descartados, bem como os mais utilizados pelos autores e que foram selecionados para representar cada parâmetro nesta pesquisa. No parâmetro habitação, Castelli et al (2009) usam percentual de domicílios sem aquecimento central no contexto da Inglaterra, o que contrasta com as pesquisas desenvolvidas no Brasil, como Mello Filho (2003) que usa domicílios com banheiro e sanitário e Keinert et al (2009) que usam domicílios em área de invasão e domicílios com dez pessoas ou mais. Para a pesquisa, optou-se pela densidade de pessoas por domicílio. 
O parâmetro saneamento não é muito recorrente por ser descartado nas pesquisas européias, tanto que em algumas discussões (Castelli et al, 2009; Arechavala, 2008) é considerado assunto já superado. Porém, nos estudos sobre qualidade de vida no Brasil e alguns países asiáticos, esses indicadores são muito presentes. Desse modo, optou-se nessa pesquisa pelo percentual de pessoas morando em domicílios com esgoto ligado na rede. Já o parâmetro saúde possui, entre os diversos indicadores, proximidade das farmácias na cidade do Porto (Martins, 2011), taxa de suicídios nos países europeus (Arechavala, 2008), sintomas de doenças e mortalidade infantil em Belo Horizonte (Nahas, 2009) e taxa de mortalidade geral na Inglaterra (Castelli et al, 2009). Para a pesquisa adotou-se uma análise de distâncias a partir das unidades de atendimento de saúde (postos de saúde e hospitais de atendimento público).

No parâmetro educação destaca-se o uso do indicador número de bibliotecas em Braila - Romênia (Sandru, 2012) e taxa de matrícula no ensino superior nos países europeus (Arechavala, 2008). No contexto da pesquisa adotou-se o percentual de pessoas com 10 anos ou mais alfabetizadas. No parâmetro transporte é possível destacar Castelli et al (2009), com pessoas que se deslocam de bicicleta ou a pé na Inglaterra, e Majumder (2007), que aponta engarrafamento, fato comum nas cidades do oriente asiático. No caso da pesquisa, adotouse análise de distâncias a partir da área central de Francisco Beltrão, onde se concentra a maior parte dos serviços públicos.

Para o parâmetro cultura pode-se destacar o acesso a internet em Braila - Romênia (Sandru, 2012), comparecimento eleitoral na Inglaterra (Castelli et al, 2009) e templos religiosos em Chittagong - Bangladesh (Majumder, 2007). Para a pesquisa, foi selecionado como indicador análise de distâncias em relação aos centros comunitários. No parâmetro esporte e lazer aponta-se viagem nas férias na Suécia (Castelli et al, 2009) e proximidades de piscinas (Martins, 2011). Para a pesquisa, adotou-se análise de distâncias em relação aos parques de caminhada e lazer.

O parâmetro infraestrutura não possui uso recorrente, sendo restrito a distribuição de energia elétrica e redes de telefonia. No contexto de Francisco Beltrão, selecionou-se como indicador o percentual de pessoas que residem em domicílios com energia elétrica da rede. No parâmetro ambiente urbano, há desde mensuração de gazes poluentes na atmosfera nos países europeus (Arechavala, 2008; Castelli et al, 2009), até análises de riscos de enchentes, deslizamentos e desmoronamentos nos contextos de cidades do Brasil e de Bangladesh (Mello Filho, 2003; Majumder, 2007; Nahas, 2009). Para a cidade de Francisco Beltrão foi usada análise de riscos de alagamentos (com base na proximidade de corpos hídricos, hipsometria e declividade).

No parâmetro trabalho e renda são usados número de desempregados na cidade do Porto (Martins, 2011), satisfação com o trabalho nos países europeus e na cidade Santo André (Arechavala, 2008; Keinert et al, 2009), pessoas que estão procurando emprego na Inglaterra (Castelli et al, 2009) e dificuldade financeira no final do mês nos países europeus (Arechavala, 2008). Para o contexto da pesquisa, optou-se pelo indicador renda média das pessoas com 10 anos ou mais ocupadas.

\section{Caracterização da Área de Estudo}

A cidade de Francisco Beltrão está na mesorregião sudoeste paranaense pela classificação do IBGE, e sua localização em relação ao estado e ao município pode ser vista na figura 1, que também apresenta a distribuição do bairros. 


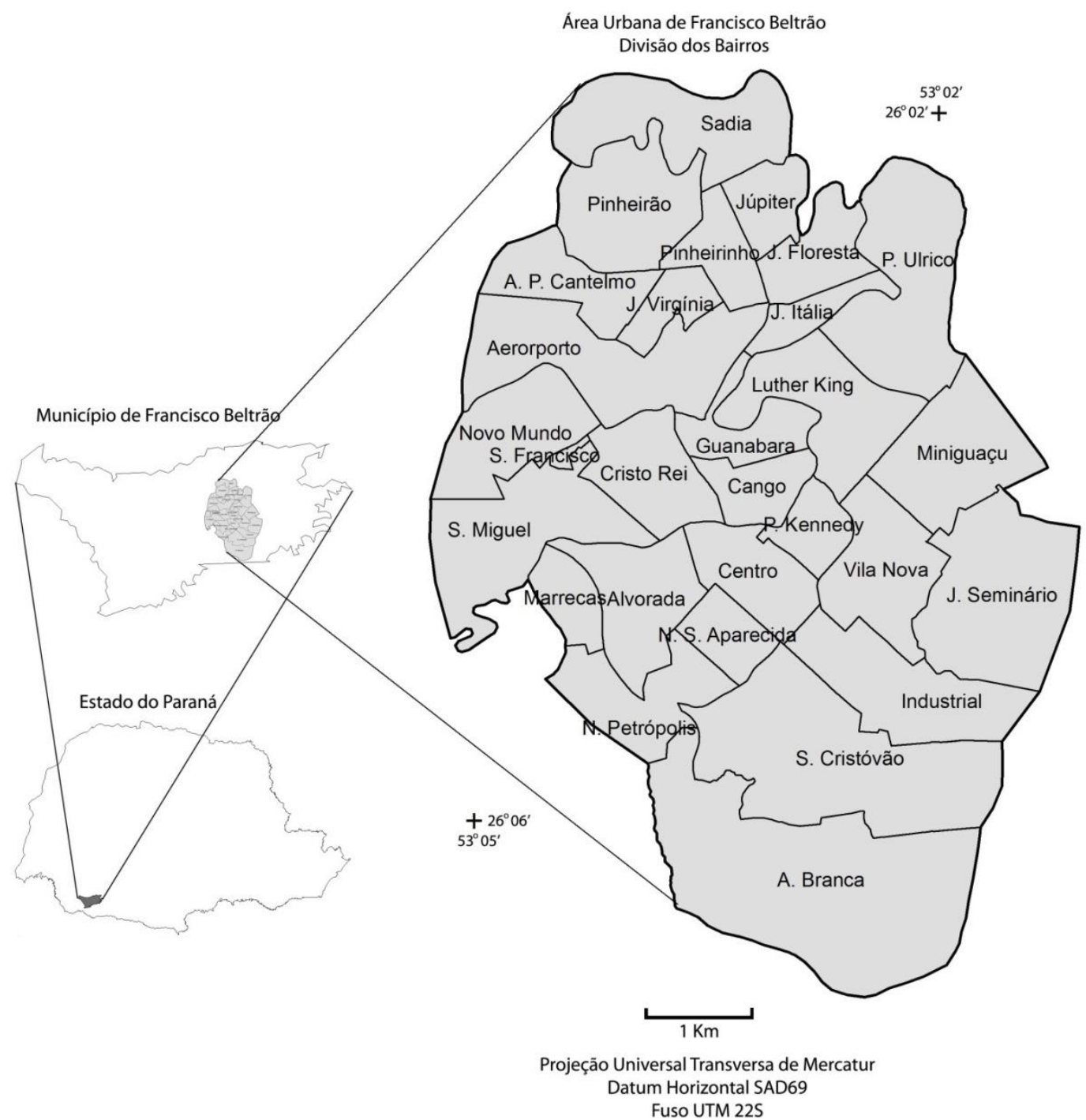

Figura 1 - Cartograma de localização da área urbana de Francisco Beltrão.

Fonte de dados: IBGE (2011).

Elaboração: O Autor (2014).

Pelos relatos históricos citados por autores como Mondardo (2009), Flavio (2011) e Machado (2009), o processo de formação da cidade se inicia no Bairro Centro na década de 1940, com intuito de suprir a demanda pelo comércio de uma região em processo de ocupação agrícola. A seguir, outros bairros vão se formando, como Cango, Nossa Senhora Aparecida, Industrial, Vila Nova, São Cristóvão e Alvorada, especialmente a partir da emancipação político-administrativa de Francisco Beltrão. Os bairros mais recentes, com exceção de alguns como o Nova Petrópolis, são formados pelo surgimento de indústrias de grande porte em determinados locais da cidade a partir da década de 1990. Esse processo distinto de formação dos bairros resulta em diferentes condições de vida da população, ocasionando uma fragmentação espacial na distribuição da qualidade de vida que se pretende identificar com o desenvolvimento desta pesquisa. 


\section{Procedimentos Metodológicos}

Os procedimentos metodológicos podem ser divididos em 4 etapas: 1) diagnóstico de indicadores a partir do Censo Demográfico 2010, 2) diagnóstico de indicadores por trabalho de campo e análise de distâncias, 3) diagnóstico dos riscos de alagamentos e 4) processamento pela somatória linear ponderada ordenada.

Para a primeira etapa, foram obtidas as tabelas com os resultados do universo e a base cartográfica com os setores censitários do estado do Paraná, uma vez que tais dados estão divididos por unidade da federação e não estão disponíveis individualmente para a cidade de Francisco Beltrão. Os setores foram ajustados espacialmente em relação a Planta Urbana fornecida pela Prefeitura Municipal, a qual é elaborada por restituição aerofotogramétrica e está em escala 1:2.000. A seguir, os dados censitários das tabelas foram geocodificados aos setores e passaram por algumas operações aritméticas para gerar 5 planos de informação: densidade de pessoas (média de pessoas por domicílio), rede de esgoto (percentual de pessoas em domicílios com esgoto ligado na rede), alfabetização (percentual de pessoas alfabetizadas com 10 anos ou mais), energia elétrica (percentual de pessoas em domicílios com energia elétrica da rede) e renda média (média de renda das pessoas com 10 anos ou mais com ocupação de trabalho). Todos os planos de informação foram divididos em classes de intervalos iguais para originar cinco níveis.

Para a segunda etapa foram buscados os endereços, no portal da Prefeitura Municipal e no Guia Paraná Sudoeste, das unidade de saúde, dos centros comunitários, dos parques e dos serviços públicos (prefeitura, câmara de vereadores, cartórios e fórum para delimitar a área central). Com os endereços, procedeu-se a localização em campo para coleta das coordenadas e georreferenciamento sobre a base cartográfica. Sobre esses dados, aplicou-se análise de distâncias com amplitude total considerando o ponto mais distante deles em relação ao perímetro urbano. Tais análises, foram divididas em cinco classes de intervalo igual para originar 4 planos de informação com cinco níveis de distâncias: unidades de saúde, área central, centros comunitários e parques.

$\mathrm{Na}$ terceira etapa, foi realizada avaliação de riscos de alagamentos a partir de uma Análise Multicritério pela somatória linear ponderada difusa, fundamentada em Delgado e Cano (2005). Para tal, foram consideradas a hipsometria, a declividade e a proximidade da rede de drenagem, com os limites máximos para alagamentos $50 \%$ acima dos valores atingidos na maior enchente dos últimos anos (abril de 2011), e com pesos definidos pela matriz de Saaty (Delgado e Cano, 2005). Como resultado foi obtido o plano de informação de alagamentos em cinco níveis (sem risco e quatro níveis de risco com intervalo igual).

$\mathrm{Na}$ quarta etapa, desenvolveu-se o processamento para sintetizar a qualidade de vida a partir dos dez planos de informação que representam os seus parâmetros, usando como metodologia Análise Multicritério pela somatória linear ponderada ordenada. $\mathrm{O}$ modelo conceitual elaborado para essa etapa pode ser visto na figura 2. 


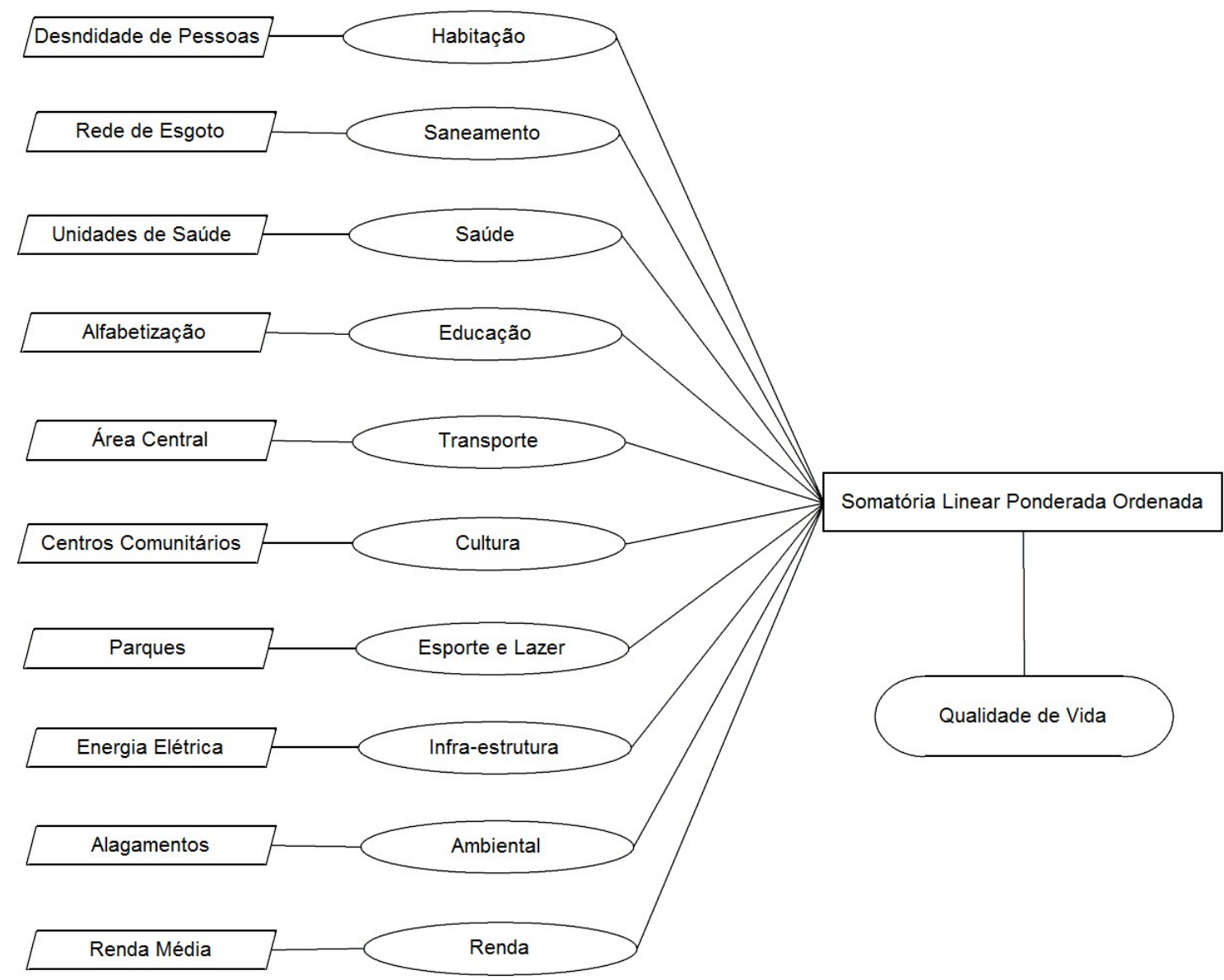

Figura 2 - Modelagem conceitual da qualidade de vida.

Fonte: O Autor (2014).

A partir do modelo, foi aplicada a equação apresentada na figura 3, onde MPn é a média ponderada, $\mathrm{P} k$ o peso atribuído aos planos de informação e $\mathrm{N} k$ a nota atribuída para as classes.

$$
\mathrm{MP}_{n}=\sum^{n} k\left[\mathrm{P}_{k}\left(\mathrm{~N}_{k}\right)\right] / \sum_{k}^{n} \mathrm{P}_{k}
$$

Figura 3 - Equação da somatória linear ponderada.

Fonte: Xavier-da-Silva (p. 77, 2001).

Na definição dos pesos para os planos de informação, foi usada como base a recorrência dos parâmetros na literatura consultada, para tal, foi calculado o percentual das frequencias de cada um deles, conforme a tabela 1. 
Tabela 1 - Frequencias e pesos dos parâmetros.

\begin{tabular}{cccc}
\hline Parâmetro & Frequencia & Peso & Peso corrigido \\
\hline Habitação & 14 & 13,1 & 13 \\
Saneamento & 8 & 7,5 & 8 \\
Saúde & 11 & 10,3 & 10 \\
Educação & 15 & 14,0 & 14 \\
Transporte & 10 & 9,3 & 9 \\
Cultura & 8 & 7,5 & 8 \\
Esporte e Lazer & 10 & 9,3 & 9 \\
Infraestrutura & 4 & 3,7 & 4 \\
Ambiente Urbano & 14 & 13,1 & 13 \\
Trabalho e Renda & 13 & 12,1 & 12 \\
\hline Total & 107 & 100 & 100 \\
\hline
\end{tabular}

Fonte: O Autor (2014).

As notas atribuídas para as classes foram de 1 a 5, obtidas a partir da metodologia proposta por Marans (2003), na qual as classes dos parâmetros e da qualidade de vida são convertidos para uma escala de cinco níveis, que pode ser interpretada como nominal (qualitativa) ou de razão (quantitativa). O quadro 2 apresenta todas as classes de cada um dos planos de informação, bem como a conversão delas para as escalas nominal e de razão.

Quadro 2 - Classes dos planos de informação e conversão para notas.

\begin{tabular}{|c|c|c|c|c|c|c|c|c|c|c|c|}
\hline $1^{*}$ & $2^{*}$ & $3^{*}$ & $4^{*}$ & $5^{*}$ & $6^{*}$ & $7^{*}$ & $8^{*}$ & $9^{*}$ & $10^{*}$ & \multicolumn{2}{|c|}{$\begin{array}{c}\text { Escalas } \\
\text { Nominal } \\
\text { e de Razão** }\end{array}$} \\
\hline 3,86 & 0 & 3.270 & 87,74 & 5.075 & 3.910 & 2.880 & 98,42 & Altíssimo & 510 & Péssima & 1 \\
3,57 & 20 & 2.616 & 90,19 & 4.060 & 3.128 & 2.304 & 98,74 & Risco & 708 & & \\
\hline 3,57 & 20 & 2.616 & 90,19 & 4.060 & 3.128 & 2.304 & 98,74 & Alto & 708 & Ruim & 2 \\
3,28 & 40 & 1.962 & 92,64 & 3.045 & 2.346 & 1.728 & 99,05 & Risco & 906 & & 3 \\
\hline 3,28 & 40 & 1.962 & 92,64 & 3.045 & 2.346 & 1.728 & 99,05 & Médio & 906 & Razoável & 3 \\
3,00 & 60 & 1.308 & 95,10 & 2.030 & 1.564 & 1.152 & 99,37 & Risco & 1.104 & & 4 \\
\hline 3,00 & 60 & 1.308 & 95,10 & 2.030 & 1.564 & 1.152 & 99,37 & Baixo & 1.104 & Boa & 4 \\
2,71 & 80 & 654 & 97,55 & 1.015 & 782 & 576 & 99,68 & Risco & 1.302 & & 5 \\
\hline 2,71 & 80 & 654 & 97,55 & 1.015 & 782 & 576 & 99,68 & Sem & 1.302 & Excelente & 5 \\
2,42 & 100 & 0 & 100 & 0 & 0 & 0 & 100 & Risco & 1.500 & & \\
\hline
\end{tabular}

*Classes dos Planos de Informação: 1 - Densidade de Pessoas (média de pessoas por domicílio); 2 - Rede de Esgoto (percentual de pessoas em domicílios com esgoto na rede); 3 Unidades de Saúde (distâncias métricas das unidades de saúde); 4 - Alfabetização (percentual de pessoas alfabetizadas com 10 anos ou mais); 5 - Área Central (distâncias métricas da área central); 6 - Centros Comunitários (distâncias métricas dos centros comunitários); 7 - Parques (distâncias métricas dos parques); 8 - Energia Elétrica (percentual de pessoas em domicílios com energia elétrica); 9 - Alagamentos (riscos de alagamentos); 10 - Trabalho e Renda (renda média em reais das pessoas ocupadas). ${ }^{* *}$ Classes convertidas a partir da proposta de Marans (2003).

Fonte: O Autor (2014). 


\section{Resultados}

Os resultados obtidos pela avaliação da qualidade de vida na cidade de Francisco Beltrão podem ser vistos na figura 4. O mapa apresenta cinco níveis da qualidade de vida segundo as classes com escala de razão propostas por Marans (2003), que podem ser convertidas para escala nominal: 1 - Péssima, 2 - Ruim, 3 - Razoável, 4 - Boa e 5 - Excelente.

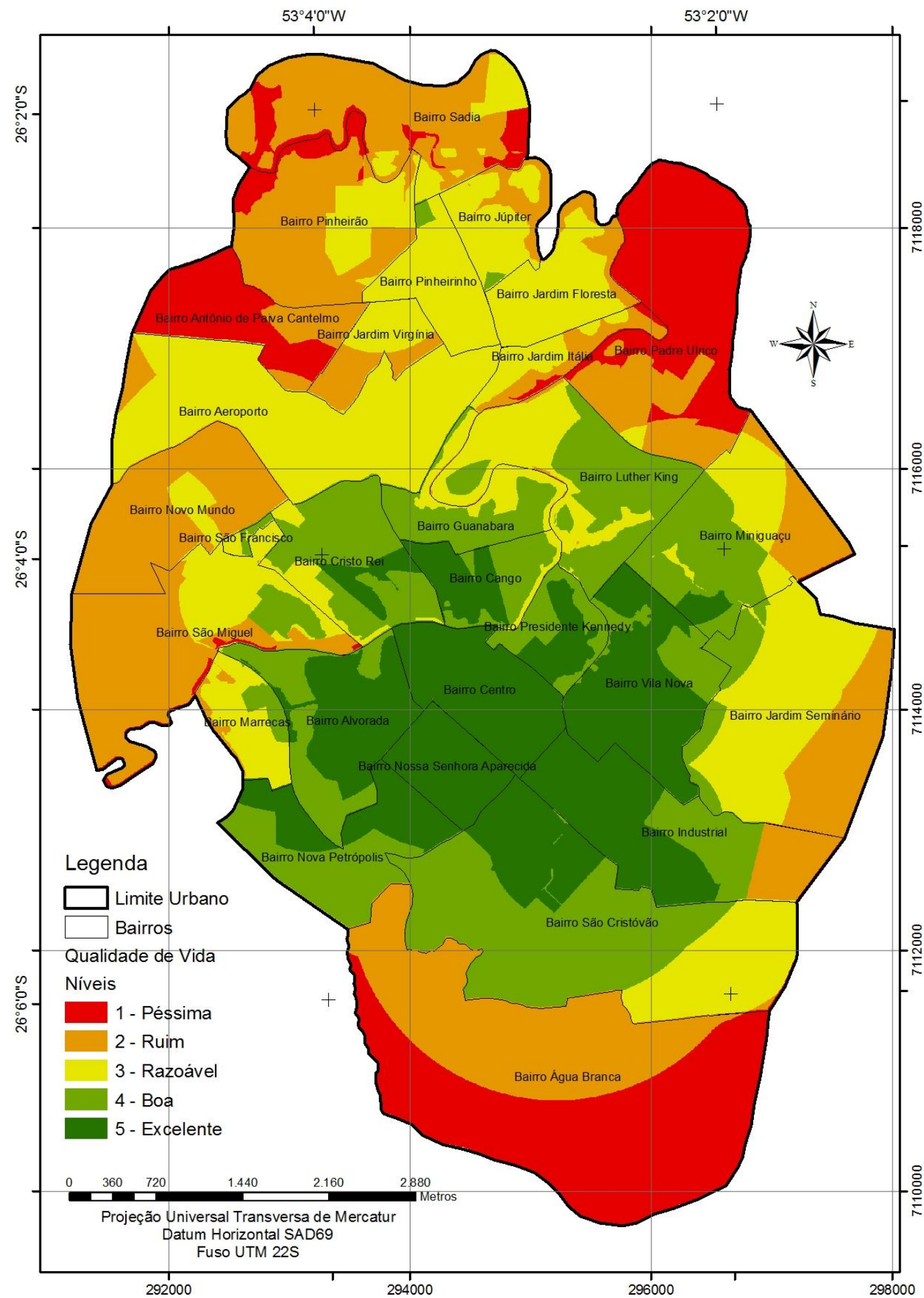

Figura 4 - Mapa da qualidade de vida na cidade de Francisco Beltrão.

Fonte: O Autor (2014). 
Os melhores níveis de qualidade de vida estão na porção central da cidade, com destaque para os bairros Centro e Nossa Senhora Aparecida, que apresentam nível "excelente" em toda a sua extensão territorial, além dos bairros Alvorada, Industrial, Vila Nova, Cango e Presidente Kennedy, onde a qualidade de vida foi diagnosticada como "boa" ou "excelente". Os fatores que mais contribuem para esses níveis elevados são: uma média de pessoas por domicílio inferior a 3,00, com exceção do Bairro Alvorada; mais de $80 \%$ das pessoas moram em domicílios com esgoto ligado na rede; os postos de saúde ficam no máximo a 1.308 m das residências; mais de 95,10\% das pessoas com dez anos ou mais são alfabetizadas; há muitos centros comunitários nesses bairros, com distâncias inferiores a 782 $\mathrm{m}$; e por estarem na área central, os principais serviços públicos ficam a menos de $1.015 \mathrm{~m}$. Os únicos parâmetros que não influenciam nas melhores condições desses bairros são: as distâncias em relação aos parques, que chegam até $2.304 \mathrm{~m}$; o percentual de pessoas em domicílios sem energia elétrica de 98,42 em alguns setores; e a renda, que varia de $\mathrm{R} \$$ 708,00 a $\mathrm{R} \$ 1.500,00$.

A qualidade de vida "razoável" à "boa" está mais presente no leste, no oeste e no centro-norte da cidade, nos bairros Miniguaçu, São Cristóvão, Luther King, Nova Petrópolis, Guanabara, São Miguel, Marrecas, Jardim Seminário, Aeroporto, Júpiter, Pinheirinho, Jardim Floresta e Cristo Rei. Os principais fatores que influenciam nesses níveis intermediários são: a média de pessoas por domicílio que varia de 2,71 a 3,57; o percentual de pessoas alfabetizadas com dez anos ou mais, que está entre 90,19 e 97,55; as distâncias dos centros comunitários, que ficam abaixo de $2.346 \mathrm{~m}$ e acima de $782 \mathrm{~m}$; e a renda média das pessoas ocupadas, que está entre $\mathrm{R} \$ 708,00$ e $\mathrm{R} \$ 1.104,00$. Já os parâmetros que não condizem com os níveis de qualidade de vida obtidos nesses bairros são: os percentuais de pessoas em domicílios com esgoto na rede, que variam de 0 a 100\%; as distâncias das unidades de saúde, que variam de 0 a 2.616 m; as distâncias dos parques, que estão entre 0 e $2.304 \mathrm{~m}$; o percentual de pessoas com energia elétrica, que está acima de 99,37 em todos os setores dos bairros; e o risco de alagamentos, que apresenta setores sem risco até de altíssimo risco.

A qualidade de vida "ruim" à "péssima" ocorre, principalmente, em bairros da porção norte da cidade (Antônio de Paiva Cantelmo, Jardim Virgínia, Pinheirão, Sadia, Jardim Itália e Padre Ulrico), mas também no Bairro Novo Mundo, à oeste, e no Bairro Água Branca, na porção sul. Os fatores que mais contribuem para esses níveis mais desfavoráveis são: a média de pessoas por domicílio, que com exceção do Bairro Água Branca, é superior a 3,00; o percentual de pessoas em domicílios com esgoto na rede, que é inferior a 40; os percentuais de pessoas alfabetizadas, que são inferiores a 92,64 na maioria dos setores; as distâncias da área central da cidade, que variam entre 2.030 e 5.075 m; a renda média das pessoas, que é inferior a $R \$ 906,00$. Já os parâmetros que menos contribuem são: as distâncias das unidades de saúde, que nos bairros da porção norte e no Novo Mundo são inferiores a 1.308 m, e no Água Branca os valores chegam a 3.270 m; as distâncias dos parques, que estão entre 0 e $2.880 \mathrm{~m}$; os percentuais de pessoas com energia elétrica da rede, que estão entre 99,05 e 100; e os riscos de alagamentos, que não existem nos bairros Novo Mundo e Água Branca, mas chegam a altíssimo risco nos bairros da porção norte.

Percebe-se, enfim, que as melhores condições de vida estão nos bairros Centro, Nossa Senhora Aparecida, Vila Nova, Industrial, Presidente Kennedy, Alvorada e Cango, que são os locais de urbanização mais antiga e concentram a maior parte do comércio e dos serviços da cidade, bem como possuem uma valorização fundiária exorbitante. Já os bairros 
que possuem as condições de vida mais desfavoráveis são Padre Ulrico, Antonio de Paiva Cantelmo, Sadia, Jardim Itália, Pinheirão, Jardim Virgínia, Novo Mundo e Água Branca, os quais possuem formação mais recente e foram constituídos por trabalhadores das linhas de produção das indústrias locais e por beneficiários de programas habitacionais.

Como recomendações, as políticas públicas devem ser priorizadas nas áreas com qualidade de vida "ruim" e "péssima", com a finalidade de diminuir as disparidades espaciais nas condições das pessoas. A partir dos indicadores analisados no decorrer da pesquisa, é possível sugerir: 1) Desenvolvimento de programas habitacionais nos bairros Padre Ulrico, Sadia, Novo Mundo e Antônio de Paiva Cantelmo; 2) Implantação de rede de esgoto em toda a porção norte da cidade e no Bairro Água Branca, ao sul; 3) Construção de unidade de saúde no Bairro Água Branca; 4) Desenvolvimento de programa de alfabetização de jovens e adultos nos bairros Padre Ulrico, Pinheirão, Antônio de Paiva Cantelmo e Novo Mundo; 5) Realocação de famílias das áreas de risco de alagamentos, especialmente dos bairros Sadia e Padre Ulrico; 6) Desenvolvimento de programas de capacitação profissional para melhoria da renda das pessoas dos bairros Padre Ulrico, Sadia, Pinheirão, Antônio de Paiva Cantelmo, Jardim Virgínia, Novo Mundo e Água Branca.

\section{Conclusões}

Há diferentes condições de vida da população na cidade de Francisco Beltrão, que resultam em contrastes espaciais na qualidade de vida entre os seus setores e os seus bairros. Tais aspectos se tornam compreensíveis apenas com análises intraurbanas, como esta apresentada, e ficam mascarados nas pesquisas que comparam unidades administrativas com viés político, tal como o Índice Firjan de Desenvolvimento Municipal (IFDM) que coloca a cidade de Francisco Beltrão como a "5a melhor do Paraná" (Pereira, 2014). Esse índice apresenta uma média geral para todo o município (rural e urbano), usando como critérios apenas dados genéricos de renda, educação e saúde, disponibilizados pelos Ministérios do Trabalho, da Educação e da Saúde.

O uso de Análise Multicritério e Geoprocessamento permitiu uma integração dos diversos aspectos que condicionam a qualidade de vida de forma sistêmica, que a partir de uma modelagem conceitual, forneceu uma visão espacial mais ampla e profunda sobre o tema na cidade de Francisco Beltrão.

\section{Referências}

ALCÁZAR, Lorena; ANDRADE, Raúl. Quality of life in urban neighborhoods in Metropolitan Lima, Peru. New York: Research Network Working Paper, 2008.

ARECHAVALA, Noelia Somarriba. Aproximación a la medición de la Calidad de Vida Social e Individual en la Europa Comunitaria. $428 \mathrm{f}$. Tese (Doutorado em Economia Aplicada) - Universidade de Valladolid, Valladolid, 2008.

ARIFWIDODO, Sigit D. Exploring the effect of compact development policy to urban quality of life in Bandung, Indonesia. City, Culture and Society, Vol. 3, n. 4, p. 303 - 311, 2012.

em: 


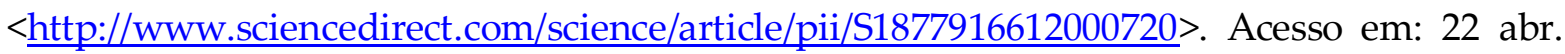
2014.

BUENO, Edir de Paiva. Dinâmica demográfica e a conformação sócio-espacial da cidade de Catalão (GO): uma análise dos níveis de desenvolvimento humano entre 1970 e 2000. 391 f. Tese (Doutorado em Geografia) - Universidade Estadual Paulista, Rio Claro, 2006.

CASTELLI, Adriana; JACOBS, Rowena; GODDARD, Maria; SMITH, Peter. Geographical Variation ins Quality of Life: the role of public service organizations. Economic and Social Research Council, London, Policy Briefing, 2009. Disponível em: $<$ http://www.york.ac.uk/media/che/documents/policybriefing/>. Acesso em: 28 maio 2012.

DELGADO, Montserrat Gómez; CANO, José Barredo. Sistemas de Información Geográfica y Evaluación Multicriterio en la Ordenación del Territorio. Madrid: RA-MA, 2005.

FITZ, Paulo Roberto. Geoprocessamento sem complicação. São Paulo: Oficina de Textos, 2008.

FLÁVIO, Luiz Carlos. Memória(s) e território elementos para o entendimento da constituição de Francisco Beltrão - PR. 386 f. Tese (Doutorado em Geografia) Universidade Estadual Paulista, Presidente Prudente, 2011.

KEINERT, Tânia Margarete Mezzomo; KEINERT, Ruben Cesar; FEFFERMENN, Marisa. Percepção da Qualidade de Vida Urbana em Santo André: resultados de uma pesquisa de opinião. In: VITTE, Claudete de Castro Silva; KEINERT, Tânia Margarete Mezzomo (Orgs.). Qualidade de Vida, Planejamento e Gestão Urbana. Rio de Janeiro: Bertrand Brasil, 2009. p. 227-264.

MACHADO, Gilnei. Transformações na Paisagem da Bacia do Rio Marrecas (SW/PR) e Perspectivas de Desenvolvimento Territorial. 284 f. Tese (Doutorado em Geografia) Universidade Estadual Paulista, Presidente Prudente, 2009.

MAJUMDER, Ahmad Kamruzzaman; HOSSAIN, Eftekhar; ISLAM, Nurul; SARWAR, Iqbal. Urban Environment Quality Mapping: a perception study on Chittagong Metropolitan City. Kathmandu University Journal of Science, Engineering and Technology, Vol. 1, n. 4, 2007. Disponível em: <http://www.nepjol.info/index.php/KUSET/article/view/2896>. Acesso em: 3 jun. 2011.

MARANS, Robert W. Understanding environmental quality through quality of life studies: the 2001 DAS and its use of subjective and objective indicators. Landscape e Urban Planning, n. 65, p. $73 \quad-\quad 83, \quad 2003 . \quad$ Disponível em: $<$ http://www.sciencedirect.com/science/article/pii/S0169204602002396>. Acesso em: 7 maio 2013.

MARQUES, Miriam Aparecida. Qualidade de Vida no Município de Macaé - RJ: análise por Geoprocessamento. 299 f. Tese (Doutorado em Geografia) - Universidade Federal do Rio de Janeiro, Rio de Janeiro, 2008.

MARTINS, Isabel Cristina Guimarães. Os Territórios da Qualidade de Vida no Porto: uma avaliação de disparidades intra-urbanas. 410 f. Tese (Doutorado em Geografia) Universidade do Porto, Porto, 2011.

MELlO FILHO, José Américo de. Qualidade de Vida na Região da Tijuca, RJ, por Geoprocessamento. 288 f. Tese (Doutorado em Geografia) - Universidade Federal do Rio de Janeiro, Rio de Janeiro, 2003. 
MONDARDO, Marcos Leandro. Os Períodos das Migrações: territórios e identidades em Francisco Beltrão-PR. 531 f. Dissertação (Mestrado em Geografia) - Universidade Federal da Grande Dourados, Dourados, 2009.

NAHAS, Maria Inês Pedrosa. Indicadores Intra-Urbanos como Instrumentos de Gestão da Qualidade de Vida Urbana em Grandes Cidades: uma discussão teórico metodológica. In: VITTE, Claudete de Castro Silva; KEINERT, Tânia Margarete Mezzomo (Orgs.). Qualidade de Vida, Planejamento e Gestão Urbana. Rio de Janeiro: Bertrand Brasil, 2009. p. 123-153.

OKTAY, Derya; RUSTEMLI, Ahmet. Measuring the of Urban Life and Neighbourhood Satisfaction: findings from Gazimagusa (Famagusta) area study. International Journal of Social Sciences and Humanity Studies, v. 2, n. 2, 2010. Disponível em: $<$ http://www.sobiad.org/ejournals/journalijss/arhieves/20102/03derya oktay.pdf $>$. Acesso em: 21/08/2012.

PEREIRA, Niomar. Pato Branco e Francisco Beltrão estão entre os melhores do Sul do Brasil. Jornal de Beltrão, Francisco Beltrão, 14 jun. 2014.

REZVANI, Mohammad Reza; MANSOURIAN, Hossain; SATTARI, Mohammad Hossain. Evaluating Quality of Life in Urban Areas (Case Study: Noorabad City, Iran). Social Indicators Research, Vol. 111, n. 3, p. 203 - 220, 2013. Disponível em: $<$ http://link.springer.com/article/10.1007\%2Fs11205-012-0048-2>. Acesso em: 18 fev. 2014.

SANDRU, Maria Iona Vlad. Quality of Life Assessment in Urban Environment Using a Geographical Informational System Model - Case Study: Brăila City. Romanian Review of Regional Studies, Vol. 8, n. 2, p. 109 - 118, 2012. Disponível em: $<$ https://www.sciencetarget.com/Journal/index.../58>. Acesso em: 19 fev. 2014.

SANTOS, Luís Delfim; MARTINS, Isabel; BRITO, Paula. O Conceito de Qualidade de Vida Urbana na Perspectiva dos Residentes na Cidade do Porto, Revista Portuguesa de Estudos Regionais, $\quad$ n. 2004.20 Disponível $<$ http://www.apdr.pt/siterper/numeros/RPER09/art01rper9.pdf $>$. Acesso em: 14 set. 2012.

SILVA-VITTE, Claudete de Castro. A Qualidade de Vida Urbana e sua Dimensão Subjetiva: uma contribuição ao debate sobre políticas públicas sobre as cidades. In: VITTE, Claudete de Castro Silva; KEINERT, Tânia Margarete Mezzomo (Orgs.). Qualidade de Vida, Planejamento e Gestão Urbana. Rio de Janeiro: Bertrand Brasil, 2009. p. 89 - 110.

STIMSON; Robert; MARANS, Robert W. Objetive Meansurement of Quality of Life Using Secondary Data Analysis. In: MARANS, Robert W.; STIMSON; Robert. Investing Quality of Urban: Theory, Methods and Empirical Research. Springer Dordrecht Heidelberg: London and New York, 2011. p. 33 - 54.

XAVIER-DA-SILVA, Jorge. Geoprocessamento para a Análise Ambiental. Rio de Janeiro: D5 Produção Gráfica, 2001. 